\title{
Vigor, floração, produção e qualidade de pêssegos ‘Chimarrita'e 'Maciel’ em função de diferentes porta-enxertos
}

\author{
Vigour, flowering, yield and quality of 'Chimarita' and 'Maciel' for different rootstocks
}

\author{
Andressa Comiotto ${ }^{\mathrm{I}}$ José Carlos Fachinello" ${ }^{\mathrm{II}}$ Alexandre Hoffmannn ${ }^{\mathrm{III}}$ Nicácia Portella Machado ${ }^{\mathrm{II}}$ \\ Simone Padilha Galarça ${ }^{I^{*}}$ Débora Leitzke Betemps ${ }^{\mathrm{II}}$
}

\section{RESUMO}

O objetivo deste trabalho foi avaliar o vigor, a floração, a produção e a qualidade de pêssegos das cultivares 'Chimarrita' e 'Maciel' sobre oito porta-enxertos. O diâmetro do tronco, acompanhamento da floração, produção $e$ qualidade de pêssegos foram os parâmetros estudados nas safras de 2008 e 2009, na Universidade Federal de Pelotas, Pelotas, RS. De acordo com os resultados obtidos, as cultivares 'Chimarrita' e 'Maciel', enxertadas sobre Umezeiro, apresentam menor vigor e sintomas de incompatibilidade quando comparadas com a enxertia nos demais porta-enxertos. A floração de ambas as cultivares foi influenciada pelos portaenxertos, podendo retardar ou antecipar o início e a plena floração em até cinco dias. A cultivar 'Maciel' enxertada sobre Tsukuba 1 foi a mais produtiva e a cultivar 'Chimarrita' apresentou maior produção quando enxertada sobre Aldrighi e Capdeboscq. O porta-enxerto Umezeiro proporcionou frutos com maior relação SS/AT para a cultivar 'Maciel' e, para 'Chimarrita', os pêssegos apresentaram maior conteúdo de sólidos solúveis.

Palavras-chave: Prunus, produtividade, crescimento, desenvolvimento, afinidade.

\section{ABSTRACT}

The objective of this research was to evaluate the vigour, flowering time, yield and quality of peach fruits from the cultivars 'Chimarrita' and 'Maciel' grafted on eight rootstocks. The experiment was carried out at the Faculdade de Agronomia Eliseu Maciel (Universidade Federal de Pelotas, Pelotas, Rio Grande do Sul, Brazil), during the 2008 and 2009 years. The obtained results showed that the 'Maciel' and 'Chimarrita' cultivars grafted on Umezeiro are less vigorous when compared to that grafted on other rootstocks, and showed signals of incompatibility. The flowering of the 'Chimarrita' and 'Maciel' peach cultivars is influenced by the rootstocks that can retard or anticipate the beginning and the full flowering in up to five days; the 'Maciel' cultivar grafted on Tsukuba 1 is the most productive and, for 'Chimarrita' the production is greater when grafted on Aldrighi and Capdeboscq. The rootstock Umezeiro provides fruit with higher SS/TA for 'Chimarrita' and 'Maciel' peach cultivars have a higher content of soluble solids.

Key words: Prunus, productivity, development, compatibility, growth.

\section{INTRODUÇÃO}

O cultivo de frutas de caroços (Prunus ssp.) possui importância econômica-social nos estados da região sul e sudeste do Brasil, principalmente no Rio Grande do Sul, responsável por mais de 60\% da produção nacional (BARBOSA et al., 2010; OLIVEIRA et al., 2011). Apesar da importância do cultivo de pêssegos e ameixas no Brasil, estudos anteriores não se preocuparam com a seleção de porta-enxertos que propiciassem desempenho produtivo superior.

Instituto Federal Rio Grande do Sul (IFRS), Campus Bento Gonçalves, Bento Gonçalves, RS, Brasil.

"Faculdade de Agronomia Eliseu Maciel (FAEM), Universidade Federal de Pelotas (UFPel), 96010-900, Pelotas, RS, Brasil. Email: sgalarca@superig.com.br. *Autor para correspondência.

IIIEmbrapa Uva e Vinho, Bento Gonçalves, RS, Brasil. 
O sucesso do cultivo dessa espécie depende da região, das práticas culturais adotadas e do porta-enxerto escolhido no momento da obtenção das mudas, sendo este último um fator que pode influenciar no desenvolvimento da cultivar, alterando a área da seção do tronco, altura, formato e crescimento da planta, volume da copa, ângulo de abertura dos ramos, nutrição da planta, potencial hídrico do xilema, fenologia, qualidade dos frutos, precocidade produtiva, produção, resistência a doenças e sobrevivência da planta (MAYER \& PEREIRA, 2006; RATO et al., 2008; REMORINI et al., 2008; NAVA et al., 2009).

A avaliação precisa das respostas agronômicas e produtivas dos porta-enxertos e a identificação da melhor combinação com cultivar copa é importante para se obter produções de qualidade (RATO et al., 2008). Sendo assim, faz-se necessário o estudo de diferentes porta-enxertos como alternativa àqueles mais utilizados na Região Sul do Brasil, a fim de se determinar combinações compatíveis com as principais cultivares copa utilizadas pelos produtores. Portanto, o objetivo do presente trabalho foi avaliar o vigor, a floração, a produção e a qualidade dos frutos de pessegueiros das cultivares 'Chimarrita' e 'Maciel' sobre oito tipos de porta-enxertos.

\section{MATERIAL E MÉTODOS}

O experimento foi realizado no pomar localizado no Centro Agropecuário da Palma, pertencente à Faculdade de Agronomia Eliseu Maciel (UFPel), no município de Pelotas, RS, com a latitude $31^{\circ} 52^{\prime} 00^{\prime \prime}$, longitude $52^{\circ} 21^{\prime} 24^{\prime \prime} \mathrm{W}$ e altitude de $13,24 \mathrm{~m}$. O clima é da categoria C e subtipo Cfa (clima subtropical), com inverno frio e úmido e verão moderado e seco. A região possui temperatura e precipitação média anual de $17,9^{\circ} \mathrm{C}$ e $1500 \mathrm{~mm}$, respectivamente. As avaliações (2008 e 2009) foram realizadas nos pessegueiros 'Chimarrita' e 'Maciel' enxertados sobre Aldrighi, Capdeboscq, Umezeiro, Flordaguard, Nemaguard, Seleção Viamão, Tsukuba 1 e Okinawa em espaçamento de 5,0x1,5m, sob sistema de condução em "V".

As variáveis avaliadas foram: diâmetro do tronco (DT, cm), volume de copa $\left(\mathrm{m}^{3}\right)$ e avaliação visual do ponto de enxertia. As flores foram contadas em cada data de avaliação, considerado o início da floração (IF) quando 10\% das flores estavam abertas, plena floração (PF) com 50\% das flores abertas e final da floração (FF) na queda das pétalas das flores. Para determinar a produção, avaliaram-se as seguintes variáveis: produção por planta (PP, kg planta $\left.{ }^{-1}\right)$ e produtividade ( $\mathrm{P}$, ton ha $\left.\mathrm{H}^{-1}\right)$. A qualidade dos frutos foi avaliada através dos sólidos solúveis (SS, ${ }^{\circ} \mathrm{Brix}$ ); acidez titulável (AT, \% de ácido málico); pH; relação SS/AT e firmeza da polpa (FP, Newtons), sendo avaliados 20 frutos por repetição.

O delineamento experimental utilizado foi o de blocos ao acaso, unifatorial. Para cada cultivar copa, foram avaliados oito porta-enxertos, com três blocos, sendo cada parcela composta por cinco plantas. Foram descartadas as plantas das extremidades de cada parcela, totalizando, assim, nove plantas úteis por parcela. Os dados obtidos foram submetidos à análise da variância, através do Teste $\mathrm{F}$ e as médias comparadas pelo teste Tukey, em que $\mathrm{P}<0,05$ através do auxílio do Programa estatístico WinStat ${ }^{\circledR}$.

\section{RESULTADOS E DISCUSSÃO}

A variável diâmetro do tronco (DT) das cultivares 'Chimarrita' e 'Maciel', em ambos os anos, foi influenciada significativamente pelos diferentes porta-enxertos, sendo que o porta-enxerto Umezeiro proporcionou menores valores para ambas as cultivares.

Em relação ao volume de copa (VC), em 2008, não houve diferença significativa para a cultivar 'Maciel', combinada com todos os porta-enxertos. Já para a cultivar 'Chimarrita', os porta-enxertos Aldrighi, Flordaguard, Nemaguard e Seleção Viamão proporcionaram maiores valores. Em 2009, os maiores valores foram encontrados na combinação 'Maciel' com Aldrighi, Flordaguard, Nemaguard, Tsukuba 1 e Seleção Viamão e, para a cultivar 'Chimarrita', o portaenxerto Umezeiro proporcionou o menor VC.

A diferença em relação ao DT e VC entre os porta-enxertos ocorre em função da maior ou menor capacidade de absorção de nutrientes do sistema radicular, da capacidade de translocação de seiva à cultivar copa e da capacidade fotossintética da cultivar copa em converter fotoassimilados em massa seca, expressa em vigor. Porta-enxertos mais vigorosos apresentam maior capacidade de absorção e translocação de água e nutrientes, e, maior produção de substâncias estimuladoras de crescimento, favorecendo o desenvolvimento da copa (PAULETTO et al., 2001). 
Pode-se considerar o Umezeiro como sendo o porta-enxerto de menor vigor dentre os avaliados em ambas as cultivares, demonstrando incompatibilidade com grau variável com a cultivar 'Chimarrita' e 'Maciel'. No entanto, é importante salientar que esse efeito ananizante, quando comparado a porta-enxertos vigorosos, pode ser uma característica interessante para o pessegueiro, se cultivado de forma adensada, conforme já relatado MAYER \& PEREIRA(2006).

No ano de 2008, os porta-enxertos Tsukuba 1 e Seleção Viamão induziram sobre 'Maciel' a maior produção por planta (PP) e a maior produtividade $(P)$, porém não diferiram de Capdeboscq, Nemaguard e Umezeiro (Tabela 1). Para a cultivar 'Chimarrita', em 2009, a produção por planta e a produtividade foram superiores em Aldrighi e Nemaguard, diferindo apenas de Umezeiro, o qual induziu a menores valores.

Os valores obtidos para a variável PP não podem ser considerados expressivos do potencial do pessegueiro, visto que as plantas ainda não tinham atingido plena produção, pois estavam em seu segundo

Tabela 1 - Características de vigor e de produção das cvs. 'Maciel' e 'Chimarrita', enxertadas sobre diferentes porta-enxertos, nos anos de 2008 e 2009. FAEM/UFPEL, 2011.

\begin{tabular}{|c|c|c|c|c|c|}
\hline Cultivar Copa & Porta-enxerto & $\mathrm{VC}^{1}\left(\mathrm{~m}^{3}\right)$ & $\mathrm{DT}(\mathrm{cm})$ & $\mathrm{PP}\left(\mathrm{kg} \mathrm{pl}^{-1}\right)$ & $\mathrm{P}\left(\right.$ ton $\left.\cdot \mathrm{ha}^{-1}\right)$ \\
\hline \multirow{8}{*}{ ‘Maciel’ 2008} & Aldrighi & $3,48^{\text {n.s. }}$ & $6,50 \mathrm{a}$ & $5,30 \mathrm{~b}$ & $7,07 \mathrm{~b}$ \\
\hline & Capdeboscq & 3,34 & $6,54 \mathrm{a}$ & $8,98 \mathrm{ab}$ & $11,97 \mathrm{ab}$ \\
\hline & Flordaguard & 3,65 & 6,63 a & $5,24 \mathrm{~b}$ & 6,99 b \\
\hline & Nemaguard & 3,55 & $6,67 \mathrm{a}$ & $6,29 \mathrm{ab}$ & $8,39 \mathrm{ab}$ \\
\hline & Okinawa & 3,29 & 6,85 a & $5,50 \mathrm{~b}$ & $7,33 \mathrm{~b}$ \\
\hline & Tsukuba 1 & 3,05 & 6,45 a & 11,39 a & 15,19 a \\
\hline & Umezeiro & 2,57 & $4,71 \mathrm{~b}$ & $8,39 \mathrm{ab}$ & $11,18 \mathrm{ab}$ \\
\hline & Seleção Viamão & 3,30 & 6,43 a & 11,32 a & $15,10 \mathrm{a}$ \\
\hline \multirow[t]{2}{*}{ CV (\%) } & & 18,12 & 7,54 & 25,18 & 25,18 \\
\hline & Aldrighi & $3,91 \mathrm{a}$ & $6,04 \mathrm{a}$ & $8,89^{\text {n.s. }}$ & $11,85^{\text {n.s. }}$ \\
\hline \multirow{7}{*}{ 'Chimarrita’ 2008} & Capdeboscq & $2,38 \mathrm{ab}$ & $6,30 \mathrm{a}$ & 10,58 & 14,10 \\
\hline & Flordaguard & 2,61 a & $6,04 \mathrm{a}$ & 6,88 & 9,17 \\
\hline & Nemaguard & 2,47 a & $5,64 \mathrm{a}$ & 5,11 & 6,81 \\
\hline & Okinawa & $2,41 \mathrm{ab}$ & 6,06 a & 5,37 & 7,15 \\
\hline & Tsukuba 1 & 2,32 ab & 5,73 a & 7,39 & 9,85 \\
\hline & Umezeiro & $1,35 \mathrm{~b}$ & $4,50 \mathrm{~b}$ & 7,90 & 10,53 \\
\hline & Seleção Viamão & 3,15 a & 6,07 a & 9,46 & 12,61 \\
\hline \multirow[t]{2}{*}{ CV (\%) } & & 15,59 & 5,80 & 25,67 & 26,67 \\
\hline & Aldrighi & 5,13 a & 8,07 a & $18,62^{\text {n.s. }}$ & $25,22^{\text {n.s. }}$ \\
\hline \multirow{6}{*}{ ‘Maciel’ 2009} & Capdeboscq & $4,50 \mathrm{ab}$ & $7,91 \mathrm{a}$ & 16,00 & 21,33 \\
\hline & Flordaguard & 5,61 a & 8,10 a & 18,63 & 24,84 \\
\hline & Nemaguard & $5,24 \mathrm{a}$ & 7,98 a & 17,88 & 23,83 \\
\hline & Okinawa & $4,68 \mathrm{ab}$ & 7,83 a & 14,74 & 19,65 \\
\hline & Tsukuba 1 & $5,74 \mathrm{a}$ & $7,64 \mathrm{a}$ & 19,60 & 26,12 \\
\hline & Umezeiro & $3,29 \mathrm{~b}$ & $5,98 \mathrm{~b}$ & 16,76 & 22,34 \\
\hline \multirow{3}{*}{ CV (\%) } & Seleção Viamão & 4,82 a & $7,64 \mathrm{a}$ & 15,79 & 21,05 \\
\hline & & 10,6 & 7,48 & 10,15 & 10,15 \\
\hline & Aldrighi & $4,71 \mathrm{a}$ & 7,16 a & $22,22 \mathrm{a}$ & 29,63 a \\
\hline \multirow{7}{*}{ 'Chimarrita’ 2009} & Capdeboscq & $4,42 \mathrm{a}$ & $7,57 \mathrm{a}$ & $20,70 \mathrm{ab}$ & $27,60 \mathrm{ab}$ \\
\hline & Flordaguard & 4,93 a & 7,68 a & $20,43 \mathrm{ab}$ & $27,24 \mathrm{ab}$ \\
\hline & Nemaguard & 4,41 a & $6,86 \mathrm{ab}$ & $22,02 \mathrm{a}$ & 29,35 a \\
\hline & Okinawa & $3,92 \mathrm{ab}$ & 7,33 a & $19,58 \mathrm{ab}$ & $26,09 a b$ \\
\hline & Tsukuba 1 & $4,13 \mathrm{ab}$ & $6,97 \mathrm{ab}$ & $19,07 \mathrm{ab}$ & $25,42 \mathrm{ab}$ \\
\hline & Umezeiro & $2,17 \mathrm{~b}$ & $5,78 \mathrm{~b}$ & $12,84 \mathrm{~b}$ & $17,11 \mathrm{~b}$ \\
\hline & Seleção Viamão & $4,22 \mathrm{a}$ & $7,14 \mathrm{a}$ & $17,84 \mathrm{ab}$ & $23,78 \mathrm{ab}$ \\
\hline CV (\%) & & 17,07 & 5,92 & 14,22 & 14,22 \\
\hline
\end{tabular}

* Médias seguidas pela mesma letra minúscula na coluna não diferem entre si pelo teste de Tukey $(\mathrm{P}<0,05)$. "n.s” = não significativo.

${ }^{1} \mathrm{VC}=$ Volume de Copa = Peso; DT = Diâmetro do Tronco ; PP= Produção por Planta ; P= Produtividade.

Ciência Rural, v.42, n.5, mai, 2012. 
(2008) e terceiro (2009) ano de produção. No entanto, o efeito do porta-enxerto já pode ser observado. STERN \& DORON (2009) relatam o efeito dos porta-enxertos na cultivar de pêra Coscia somente a partir do $4^{\circ}$ ano de produção, expandindo-se ano a ano, verificando diferenças consideráveis e significativas no 9o ano de avaliação.

Ao contrário da literatura, a qual descreve porta-enxertos menos vigorosos e anões como produtivos, conforme constatado por PICOLOTTO et al. (2009) ao avaliar a cultivar 'Chimarrita' sobre o portaenxerto GF 305, no presente trabalho, não foram observados esses resultados para Umezeiro, quando enxertado sob as cultivares 'Chimarrita’ e 'Maciel'.

A baixa produção por planta e produtividade observada em 'Chimarrita' quando enxertada sobre Umezeiro, em 2009 (Tabela 1), quando comparada aos demais porta-enxertos, provavelmente tenha ocorrido em função da falta de afinidade que ocorre entre este porta-enxerto e a cultivar copa e a certo grau de incompatibilidade que impede ou limita a absorção e translocação de água, sais minerais e carboidratos disponíveis, sendo, este último, responsável pela formação das gemas floríferas.

De acordo com a observação visual, atribuise a menor produção por planta observada em ‘Chimarrita', quando comparada à 'Maciel', devido às suas características genéticas e à incompatibilidade com Umezeiro, traduzindo-se numa diminuição da produção por planta.

Avaliando-se visualmente o ponto de união entre as cultivares copa e os porta-enxertos a campo, observou-se que Umezeiro, quando enxertado sobre 'Chimarrita' e 'Maciel', apresentou anomalias no ponto de enxertia na forma de intumescimento bastante visível. No entanto, os sintomas morfológicos externos da planta como hipertrofia do ponto de enxertia e decréscimo na altura do diâmetro do tronco e no volume de copa da planta foram muito mais acentuados para a cultivar 'Chimarrita'.

Houve alteração da floração do pessegueiro em função dos porta-enxertos nos diferentes estádios de avaliação (Tabela 2). O início de floração (IF) foi antecipado em sete dias na cultivar 'Maciel' sobre a Seleção Viamão em 2008 e, em dois dias, em 2009, quando enxertado sobre o porta-enxerto Okinawa. Em relação à plena floração (PF) e final de floração (FF), verificam-se variações com intervalos menores.

Para a 'Chimarrita', o IF ocorreu em períodos diferentes de ano para ano e os intervalos de floração entre os porta-enxertos foram mais curtos quando comparadas com a cultivar 'Maciel' (Tabela 2). Em relação à PF e FF, os resultados foram semelhantes aos obtidos para a cultivar 'Maciel'.

Tabela 2 - Início, plena e final da floração das cvs. 'Maciel' e 'Chimarrita', enxertadas sobre diferentes porta-enxertos. FAEM/UFPEL, 2011.

\begin{tabular}{|c|c|c|c|c|c|c|c|}
\hline \multirow{2}{*}{ Cultivar } & \multirow{2}{*}{ Porta-enxerto } & \multicolumn{2}{|c|}{ Início da floração (IF) } & \multicolumn{2}{|c|}{ Plena floração (PF) } & \multicolumn{2}{|c|}{ Final da floração (FF) } \\
\hline & & 2008 & 2009 & 2008 & 2009 & 2008 & 2009 \\
\hline \multirow{8}{*}{ ‘Maciel’ } & Aldrighi & $13 / 07$ & $04 / 08$ & $25 / 07$ & $10 / 08$ & $25 / 07$ & $16 / 08$ \\
\hline & Capdeboscq & $12 / 07$ & 03/08 & $23 / 07$ & $11 / 08$ & $23 / 07$ & $16 / 08$ \\
\hline & Flordaguard & $18 / 07$ & $04 / 08$ & $25 / 07$ & $15 / 08$ & $25 / 07$ & $16 / 08$ \\
\hline & Nemaguard & $15 / 07$ & 03/08 & $25 / 07$ & $10 / 08$ & $25 / 07$ & $17 / 08$ \\
\hline & Okinawa & $17 / 07$ & 05/08 & $26 / 07$ & $13 / 08$ & $26 / 07$ & $17 / 08$ \\
\hline & Tsukuba 1 & $16 / 07$ & $04 / 08$ & $24 / 07$ & $12 / 08$ & $24 / 07$ & $17 / 08$ \\
\hline & Umezeiro & $14 / 07$ & 05/08 & $21 / 07$ & $12 / 08$ & $21 / 07$ & $17 / 08$ \\
\hline & Seleção Viamão & $10 / 07$ & 03/08 & $25 / 07$ & $11 / 08$ & $25 / 07$ & $17 / 08$ \\
\hline \multirow{8}{*}{ 'Chimarrita' } & Aldrighi & $22 / 07$ & 06/08 & 03/08 & $14 / 08$ & $12 / 08$ & $17 / 08$ \\
\hline & Capdeboscq & $21 / 07$ & $10 / 08$ & $02 / 08$ & $15 / 08$ & $11 / 08$ & $17 / 08$ \\
\hline & Flordaguard & $21 / 07$ & $10 / 08$ & 03/08 & $15 / 08$ & $11 / 08$ & $17 / 08$ \\
\hline & Nemaguard & $19 / 07$ & 08/08 & 01/08 & $14 / 08$ & $11 / 08$ & $17 / 08$ \\
\hline & Okinawa & $22 / 07$ & $09 / 08$ & 03/08 & $14 / 08$ & $11 / 08$ & $17 / 08$ \\
\hline & Tsukuba 1 & $23 / 07$ & $11 / 08$ & 03/08 & $15 / 08$ & $12 / 08$ & $17 / 08$ \\
\hline & Umezeiro & $23 / 07$ & $10 / 08$ & $04 / 08$ & $15 / 08$ & $13 / 08$ & $17 / 08$ \\
\hline & Seleção Viamão & $20 / 07$ & 09/08 & $02 / 08$ & $14 / 08$ & $10 / 08$ & $17 / 08$ \\
\hline
\end{tabular}


Provavelmente, o retardo no IF da cultivar 'Maciel' sobre 'Flordaguard' e 'Okinawa' em 2008, da $\mathrm{PF}$, quando enxertada sobre Okinawa (2008) e Flordaguard (2009) e a antecipação do IF, quando enxertada sobre Nemaguard (2009), Seleção Viamão e Capdeboscq (2008 e 2009) e antecipação da PF quando enxertada sobre Nemaguard (2009), sejam em função do vigor destes porta-enxertos. Para a cultivar 'Chimarrita', a antecipação do IF foi verificada quando enxertada sobre Seleção Viamão (2008), Aldrighi (2009) e Nemaguard (2008 e 2009) e a antecipação da PF foi observada quando enxertada sobre Nemaguard (2008 e 2009) e Seleção Viamão (2008 e 2009). Observou-se retardamento no IF para 'Chimarrita' enxertada sobre Umezeiro (2008 e 2009) e, também, na PF (2008). A influência dos porta-enxertos sobre a floração também foi constatada por PICOLOTTO et al. (2009). Os dados obtidos neste trabalho corroboram MOGHADAM \& MOKHTARIAN (2007), que observaram o atraso no florescimento de pessegueiros enxertados sobre Siberia $\mathrm{C}$ em quatro a cinco dias.

A época de floração depende de vários fatores como o vigor do porta-enxerto, da cultivar copa, das condições edafoclimáticas, dos problemas fitossanitários, do manejo do pomar e da época de poda da planta. Considera-se que as diferenças entre portaenxertos e condições climáticas são fortes e complexas, com variações significativas ano a ano (DAZA et al., 2008).

Os frutos da cultivar 'Maciel' enxertada sobre Umezeiro, produzidos no ano de 2008, apresentaram menor acidez titulável (AT), diferindo apenas de Aldrighi e Nemaguard; maior relação SS/AT, não diferindo de Tsukuba 1 e pH mais elevado dentre os porta-enxertos avaliados (Tabela 2). Quanto à firmeza da polpa (FP), Aldrighi induziu frutos com uma resistência superior, porém diferiu apenas de Seleção Viamão que induziu menor FP dos frutos. No ano de 2009, não houve diferença significativa para as variáveis físico-químicas avaliadas.

Para 'Chimarrita', em 2008, o maior teor de SS nos frutos foi induzido pelo porta-enxerto Umezeiro, embora diferindo significativamente apenas de Seleção Viamão, com menor teor. Esse resultado corrobora CANTÍN et al. (2010), que também observaram o maior teor de SS em frutos de cereja da cultivar Van, enxertada sobre o porta-enxerto ananizante Gisela 5. Provavelmente, o Umezeiro, por ser um porta-enxerto menos vigoroso, permite a passagem de maior luminosidade através da copa da planta. Segundo
PICOLOTTO et al. (2009), o teor de SS dos pêssegos recebe maior influência de alguns fatores, tais como posição do fruto na planta, penetração de luz na copa, tipo de ramo e de poda do que pelo porta-enxerto. Para as demais variáveis avaliadas, não houve diferença significativa.

Em 2009, os frutos da cultivar 'Chimarrita', enxertada sobre Seleção Viamão, apresentaram pH mais elevado, porém, diferindo somente de Aldrighi (Tabela 3). Avaliando-se a FP, obtiveram-se valores significativamente superiores para Aldrighi, porém diferiu apenas de Umezeiro e Okinawa. Não houve diferença significativa para as demais variáveis físicoquímicas avaliadas.

Os resultados obtidos para a variável FP dos frutos das cultivares 'Chimarrita' e 'Maciel', nos anos de 2008 e 2009, estiveram abaixo dos valores descritos por PICOLOTTO et al. (2009) ao estudarem essa característica para as mesmas cultivares do presente estudo.

A relação SS/AT é uma das melhores formas de avaliação do sabor de uma fruta e é usualmente utilizada para avaliar o seu grau de maturação. Portanto, o maior valor encontrado em frutos de 'Maciel' pelo porta-enxerto Umezeiro, em 2008, parece ser um indicativo de antecipação da maturação. GIORGI et al. (2005) observaram resultados semelhantes com portaenxertos pouco vigorosos, em que a cultivar 'Suncrest', enxertada sobre GF 677, produziu frutos de menor tamanho, menor AT e maior relação SS/AT. MEREDIT et al. (1989) relatam que, para pêssegos serem considerados de boa qualidade, essa relação deve ser maior ou igual a 15, o que foi constatado para as duas cultivares no ano de 2008 e para 'Chimarrita', avaliada em 2009.

\section{CONCLUSÃO}

As cultivares 'Maciel' e 'Chimarrita' sobre 'Umezeiro' são menos vigorosas quando comparadas com os demais porta-enxertos e apresentam sintomas de incompatibilidade.

A época de floração e a qualidade dos pêssegos ‘Maciel' e 'Chimarrita' é influenciada pelos porta-enxertos, podendo retardar ou antecipar o início da floração em até oito dias.

A cultivar 'Maciel' sobre o porta-enxerto Tsukuba 1 é mais produtiva e, para a cultivar 'Chimarrita', a produção é maior, quando enxertada sobre Aldrighi e Capdeboscq. 
Vigor, floração, produção e qualidade de pêssegos 'Chimarrita' e 'Maciel’ em função de diferentes porta-enxertos.

Tabela 3 - Sólidos solúveis (SS), acidez titulável (AT), relação SS/AT, pH e firmeza da polpa (FP) de pêssegos 'Maciel’ e 'Chimarrita', sobre oito porta-enxertos. FAEM/UFPEL, 2011.

\begin{tabular}{|c|c|c|c|c|c|}
\hline Porta-enxertos & SS ( ${ }^{\circ}$ Brix) & AT (\% ácido málico) & SS/AT & $\mathrm{pH}$ & Firmeza da polpa $(\mathrm{N})$ \\
\hline Aldrighi ${ }^{(1)}$ & $11,91^{\text {ns }}$ & $0,70 \mathrm{a}$ & $16,59 \mathrm{c}$ & 3,63cd & 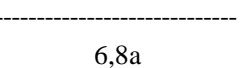 \\
\hline Capdeboscq & 12,53 & 0,66ab & $18,32 b c$ & 3,64bcd & 4,88ab \\
\hline Flordaguard & 12,06 & 0,66ab & $17,69 b c$ & 3,67abcd & 4,44ab \\
\hline Nemaguard & 12,36 & $0,70 \mathrm{a}$ & $17,07 \mathrm{bc}$ & $3,57 d$ & 5,13ab \\
\hline Okinawa & 12,28 & 0,63ab & $18,80 \mathrm{bc}$ & 3,76ab & 5,71ab \\
\hline Tsukuba 1 & 12,53 & 0,60ab & $20,00 a b$ & 3,71abc & 4,89ab \\
\hline Umezeiro & 12,88 & $0,60 \mathrm{~b}$ & $20,68 a$ & $3,78 a$ & 5,71ab \\
\hline Seleção Viamão & 12,23 & 0,65ab & $18,07 \mathrm{bc}$ & 3,63cd & $4,24 b$ \\
\hline CV (\%) & 4,81 & 5,37 & 5,45 & 1,22 & 16,38 \\
\hline Aldrighi $^{(2)}$ & 11,36ab & $0,35^{\mathrm{ns}}$ & $31,86^{\mathrm{ns}}$ & $4,20^{\mathrm{ns}}$ & $10,92^{\mathrm{ns}}$ \\
\hline Capdeboscq & $12,24 \mathrm{ab}$ & 0,48 & 27,93 & 4,08 & 6,67 \\
\hline Flordaguard & 11,89ab & 0,33 & 35,71 & 4,30 & 8,43 \\
\hline Nemaguard & 11,33ab & 0,30 & 37,12 & 4,43 & 8,35 \\
\hline Okinawa & 11,49ab & 0,38 & 30,06 & 4,18 & 9,49 \\
\hline Tsukuba 1 & 11,03ab & 0,38 & 28,68 & 4,20 & 7,91 \\
\hline Umezeiro & 13,03a & 0,34 & 37,87 & 4,30 & 8,18 \\
\hline Seleção Viamão & $10,76 \mathrm{~b}$ & 0,32 & 33,09 & 4,19 & 8,56 \\
\hline CV (\%) & 6,29 & 25,56 & 13,90 & 3,60 & 21,34 \\
\hline Aldrighi $^{(1)}$ & $9,20^{\mathrm{ns}}$ & $0,84^{\mathrm{ns}}$ & $11,08^{\mathrm{ns}}$ & $3,58^{\text {ns }}$ & $6,56^{\mathrm{ns}}$ \\
\hline Capdeboscq & 9,49 & 0,79 & 12,13 & 3,62 & 6,99 \\
\hline Flordaguard & 8,70 & 0,84 & 10,45 & 3,61 & 7,28 \\
\hline Nemaguard & 9,16 & 0,85 & 10,79 & 3,53 & 6,33 \\
\hline Okinawa & 9,69 & 0,84 & 11,88 & 3,58 & 6,95 \\
\hline Tsukuba 1 & 9,84 & 0,81 & 12,49 & 3,63 & 6,34 \\
\hline Umezeiro & 9,94 & 0,76 & 13,15 & 3,60 & 6,91 \\
\hline Seleção Viamão & 10,24 & 0,82 & 12,55 & 3,52 & 6,54 \\
\hline CV (\%) & 13,30 & 7,95 & 19,84 & 1,96 & 12,64 \\
\hline Aldrighi $^{(2)}$ & $9,06^{\mathrm{ns}}$ & $0,45^{\mathrm{ns}}$ & $20,01^{\mathrm{ns}}$ & $4,41 \mathrm{c}$ & $7,68 \mathrm{a}$ \\
\hline Capdeboscq & 9,18 & 0,45 & 20,48 & $4,53 b c$ & $5,11 \mathrm{ab}$ \\
\hline Flordaguard & 8,74 & 0,42 & 20,94 & 4,46bc & 5,50ab \\
\hline Nemaguard & 8,50 & 0,45 & 19,05 & 4,61bc & $6,00 \mathrm{ab}$ \\
\hline Okinawa & 9,55 & 0,46 & 20,84 & $4,72 b c$ & $4,38 b$ \\
\hline Tsukuba 1 & 9,20 & 0,41 & 22,79 & $4,49 b c$ & $5,00 \mathrm{ab}$ \\
\hline Umezeiro & 9,33 & 0,36 & 26,52 & $4,70 \mathrm{bc}$ & $3,05 b$ \\
\hline Seleção Viamão & 10,17 & 0,42 & 24,24 & $5,03 a$ & 5,11ab \\
\hline CV (\%) & 8,12 & 9,25 & 11,92 & 2,06 & 20,62 \\
\hline
\end{tabular}

*Medias seguidas pela mesma letra minúscula na coluna não diferem entre si pelo teste de Tukey $(\mathrm{P}<0,05)$. "n.s.” = não significativo

${ }^{(1)}$ referente à cultivar 'Maciel' e ${ }^{(2)}$ referente à cultivar 'Chimarrita'.

\section{REFERÊNCIAS}

BARBOSA, W. et al. Advances in low-chilling peach breeding at Instituto Agronômico, São Paulo State, Brazil. Acta Horticulturae, v.872, p.147-150, 2010.

CANTÍN, C.M. et al. Growth, yield and fruit quality of 'Van' and 'Stark Hardy Giant' sweet cherry cultivars as influenced by grafting on different rootstocks. Scientia Horticulturae, v.123 p.329-335, 2010. Disponível em: <http:// w w w.sciencedirect.com/science/article / pi i / S0304423809004506>. Acesso em: 16 maio, 2011. doi:10.1016/j.scienta.2009.09.016.

DAZA, A. et al. Fruit quality parameters of 'Pioneer' Japanese plums produced on eight different rootstocks. Scientia Horticulturae, v.118, n.3, p.206-211, 2008. Disponível em: <http://www.sciencedirect.com/science/article/pii/ S0304423808002264>. Acesso em: 12 jan. 2011. doi:10.1016/ j.scienta.2008.06.003.

Ciência Rural, v.42, n.5, mai, 2012. 
GIORGI, M. et al. The rootstock effects on plant adaptability, production, fruit quality, and nutrition in the peach (cv. 'Suncrest'). Scientia Horticulturae, v.107, p.36-42, 2005. Disponível em: <http://www.sciencedirect.com/science/article/ pii/S0304423805002086>. Acesso em: 30 nov.2010. doi:10.1016/j.scienta.2005.06.003.

MAYER, N.A.; PEREIRA, F.M. Vigor de clones de umezeiro e pessegueiro 'Okinawa' propagados por estacas herbáceas. Pesquisa Agropecuária Brasileira, v.41, n.5, p.883-887, 2006. Disponível em: <http://webnotes.sct.embrapa.br/pab/ pab.nsf/FrAnual>. Acesso em: 9 nov. 2011. doi: dx.doi.org/ 10.1590/S0100-204X2006000500024.

MOGHADAM, E.G.; MOKHTARIAN, A. Evaluation of the effects of plum rootstocks on time of flowering in apricot ('Shahroudi' and 'Lasgerdi' cultivars) trees. Acta Horticulturae, v.734, p.163-168, 2007.

NAVA, G.A. et al. Reprodução do pessegueiro: efeito genético, ambiental e de manejo das plantas. Revista Brasileira Fruticultura, v.31, n.4, p.1218-1233, 2009. Disponível em: <http://www.scielo.br/scielo.php?script=sci_arttext\&pid $=$ S0100-29452009000400042\&lng=pt\&nrm=iso\&tlng=pt $>$. Acesso em: 9 nov. 2011. doi: dx.doi.org/10.1590/S010029452009000400042.

OLIVEIRA, M.C. et al. Seleção de ameixeiras promissoras para a Serra da Mantiqueira. Revista Ceres, v.58, n.4, p.531535, 2011. Disponível em: <http://www.scielo.br/ scielo.php ? script = sci_art text \& pid=S 0034 $737 X 2011000400019 \& \operatorname{lng}=p t \& n r m=i s o \& t \operatorname{lng}=p t>$. Acesso em: 11 mar. 2011. doi: dx.doi.org/10.1590/S0034737 X2011000400019.
PAULETTO, D. et al. Produção e vigor da videira 'Niágara Rosada' relacionados com o porta-enxerto. Pesquisa Agropecuária Brasileira, v.36, n.1, p.115-121, 2001. Disponível em:< http://webnotes.sct.embrapa.br/pab/pab.nsf/ FrAnual. Acesso em: 04 nov. 2011. doi: dx.doi.org/10.1590/ S0100-204X2001000100014.

PICOLOTTO, L. et al. Características vegetativas, fenológicas e produtivas do pessegueiro cultivar Chimarrita enxertado em diferentes porta-enxertos. Pesquisa Agropecuária Brasileira, v.44, n.6, p.583-589, 2009. Disponível em: <http:/ /www.scielo.br/scielo.php?script=sci_arttext \&pid= S0100204X2009000600006\&lng $=$ pt $\& n r m=$ iso\&tlng $=p t>$. Acesso em: 9 nov. 2011. doi: dx.doi.org/10.1590/S0100204 X2009000600006.

RATO, A.E. et al. Soil and rootstock influence on fruit quality of plums (Prunus domestica L.). Scientia Horticulturae, v.118, p.218-222, 2008. Disponível em: <http:// w w w.sciencedirect.com/science/article/pi i/ S030442380800229X $>$. Acesso em: 09 nov. 2011. doi: 10.1016/j.scienta.2008.06.013.

REMORINI, D. et al. Effect of rootstocks and harvesting time on the nutritional quality of peel and flesh of peach fruits. Food Chemistry, v.110, p.361-367, 2008. Disponível em: <http://www.sciencedirect.com/science/article/pii/ S030881460800174X $>$. Acesso em: 09 nov. 2011. doi:10.1016/j.foodchem.2008.02.011.

STERN, R.A.; DORON, I. Performance of 'Coscia' pear (Pyrus communis) on nine rootstocks in the north of Israel. Scientia Horticulturae, v.119, n.3, p.252-256, 2009. Disponível em: <http://www.sciencedirect.com/science/article/pii/ S0304423808003300>. Acesso em: 09 nov. 2011. doi: 10.1016/j.scienta.2008.08.002. 